\title{
ORIGINALES
}

\section{Efectos de la comunicación integrada de marketing sobre el valor de marca: el rol de la cocreación de valor y el conocimiento ecológico del huésped*}

\author{
MARIIA BORDIAN** \\ IRENE GIL-SAURA***

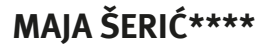

\footnotetext{
* El presente artículo se ha desarrollado en el marco del proyecto de investigación financiado por la Agencia Estatal de Investigación del Ministerio de Ciencia e Innovación (Referencia: PID2020-11266oRB-loo) y de la Ayuda para Acciones Especiales de Investigación de la Universitat de València (Referencia: UV-INV-AE-1553911).

** Doctoranda en Marketing. Universidad de Valencia, Valencia, España. E-mail: mariia.bordian@uv.es. ORCID: 0000-0002-5505-0780. Google Scholar: https://scholar.google.com.ua/citations?user=ocfypvEAAAAJ\&hl=en.

*** Doctora en Ciencias Económicas y Empresariales. Universidad de Valencia, Valencia, España. E-mail: irene.gil@uv.es. ORCID: 0000-0002-5758-0806. Google Scholar: https://scholar.google.com.ua/citations?user=5Qh81MYAAAAJ\&hl=uk\&oi=sra. Scopus Author ID: https://www.scopus.com/authid/detail.uri?authorld=26430896600.

$\star \star \star \star$ Doctora en Marketing. Universidad de Valencia, Valencia, España. E-mail: maja.šerić@uv.es. ORCID: 00oo-0oo2-7799-2592. Google Scholar: https://scholar.google.com.ua/citations?user=KaS2Q8EAAAAJ\&hl=uk. Scopus Author ID: https://www.scopus.com/ authid/detail.uri?authorld=6508027282.
} 


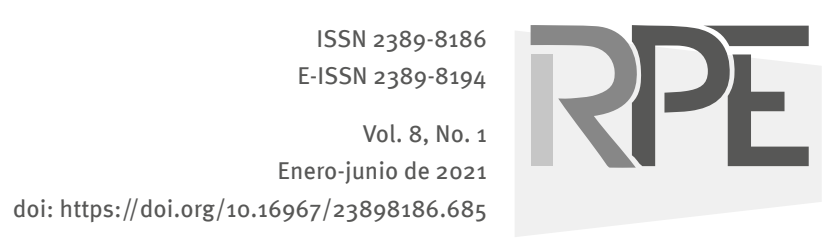

COMO CITAR ESTE ARTÍCULO

How to cite this article:

Bordian, M., Gil-Saura, I. y Šerić, M. (2021). Efectos de la comunicación integrada de marketing sobre el valor de marca: el rol de la cocreación de valory el conocimiento ecológico del huésped. Revista Perspectiva Empresarial, 8(1), 55-70.

Recibido: 07 noviembre de 2020 Aceptado: 02 de marzo de 2021
RESUMEN Objetivo. Examinar la influencia de la comunicación integrada de marketing para la sostenibilidad en la cocreación de valor y en el valor de marca de hotel a la luz del papel mediador que esta pudiera desempeñar. Además se observó cómo el conocimiento ecológico del huésped puede actuar intensificando o no dichos efectos. Metodología. El estudio empírico se realizó entre 303 huéspedes de hoteles ubicados en Ucrania, en 2018. Resultados. Los resultados revelan que la comunicación integrada de marketing tiene una influencia significativa en la cocreación de valor y en valor de marca de hotel, afirmándose el rol mediador parcial de la cocreación en dicha relación. Conclusiones. Se evidencia que el conocimiento ecológico del huésped modera la relación entre la comunicación integrada de marketing y el valor de marca de hotel, mostrando que los huéspedes con bajo nivel de conocimiento ecológico son más sensibles al mensaje sobre la sostenibilidad; intensificándose, en este caso, el efecto de la comunicación integrada de marketing sobre el valor de marca.

PALABRAS CLAVE comunicación integrada de marketing, conocimiento ecológico, cocreación de valor, valor de marca, industria hotelera.

\section{Effects of integrated marketing communication on brand equity: the role of value co-creation and guest's ecological knowledge}

ABSTRACT Objective. To examine the influence of integrated marketing communication for sustainability on value co-creation and hotel brand equity in light of the mediating role it may play. In addition, it was observed how guests' ecological knowledge can intensify, or not, such effects. Methodology. The empirical study was conducted among 303 guests of hotels located in Ukraine, in 2018. Results. The results reveal that integrated marketing communication has a significant influence on value co-creation and hotel brand equity, confirming the partial mediating role of co-creation in such relationship. Conclusions. It is evidenced that guests' ecological knowledge moderates the relationship between integrated marketing communication and hotel brand equity, showing that guests with low level of ecological knowledge are more sensitive to the message about sustainability; intensifying, in this case, the effect of integrated marketing communication on brand equity.

KEYWORDS Integrated marketing communication, green knowledge, value co-creation, brand equity, hotel industry. 


\section{Efeitos da comunicação integrada de marketing no valor da marca: 0 papel da cocriação de valor e do conhecimento ecológico do hóspede}

RESUMO Objetivo. Examine a influência da comunicação de marketing integrada para a sustentabilidade na cocriação de valor e no valor da marca do hotel à luz do papel de mediação que pode desempenhar. Além disso, observou-se como o conhecimento ecológico do hospedeiro pode atuar intensificando ou não esses efeitos. Metodologia. O estudo empírico foi realizado com 303 hóspedes de hotéis localizados na Ucrânia, em 2018. Resultados. Os resultados revelam que a comunicação integrada de marketing tem uma influência significativa na cocriação de valor e no valor da marca hoteleira, afirmando o papel mediador parcial da cocriação nessa relação. Conclusões. Constatase que o conhecimento ecológico do hóspede modera a relação entre a comunicação de marketing integrada e o valor da marca hoteleira, mostrando que os hóspedes com baixo nível de conhecimento ecológico são mais sensíveis à mensagem sobre sustentabilidade; intensificando, neste caso, o efeito da comunicação integrada de marketing no valor da marca.

PALAVRAS CHAVE comunicação integrada de marketing, conhecimento ecológico, cocriação de valor, valor da marca, indústria hoteleira. 


\section{Introducción}

La industria del turismo ha sido identificada como un contribuyente importante en las emisiones degases de efecto invernadero; su efecto representa alrededor del $8 \%$ de las emisiones mundiales de dióxido de carbono (Lenzen et al., 2018). Reconociendo esta realidad, muchos hoteles han comenzado a "tomar consciencia ecológica" al incorporar prácticas respetuosas con el medio ambiente para minimizar las emisiones de carbono y la contaminación (Moise et al., 2019; Moise, Gil-Saura and Ruiz-Molina, 2020).

El sector hotelero en Ucrania ha empezado a familiarizarse con la necesidad de implementar prácticas sostenibles e incluso algunos hoteles de cadenas internacionales recientemente están siendo reconocidos con algún tipo de sello o certificación ecológica. Pero como se evidencia en la literatura relacionada con la gestión hotelera, la falta de comprensión mutua entre el personal del hotel y el huésped sobre la importancia de llevar a cabo y colaborar en dichas prácticas supone un claro freno a su implementación (Tölkes, 2018). Una de las razones de este fenómeno es el uso ineficiente de las herramientas de comunicación, unida a la falta de competencia técnica por parte de las empresas (Gössling and Buckley, 2016; Wehrli et al., 2014; Tölkes, 2018); ello genera la necesidad de analizar la eficacia de la comunicación hotelera desde una perspectiva de sostenibilidad medioambiental. Este artículo investiga la comunicación para la sostenibilidad de los hoteles a través del enfoque de la comunicación integrada de marketing - CIM-, centrándose en el efecto sinergia que aporta la persuasión total de dos o más elementos de comunicación integrados (Kim, Kim and Marshall, 2016).

Por otra parte la cocreación de valor y el valor de marca se consideran variables significativas para la empresa hotelera, proporcionando ventajas competitivas exclusivas para la empresa. La cocreación de valor a través de la contribución y la colaboración puede (i) causar una mejor aceptación de las prácticas ecológicas de la empresa por parte de los turistas (Gössling et al., 2009; Arnold, 2017; Chang, 2019) y (ii) mejorar las ventajas competitivas del hotel, aumentando su valor de marca (González-
Mansilla, Berenguer-Contrí and Serra-Cantallops, 2019). Sin embargo todavía existe la necesidad de ampliar la escasa investigación sobre la relación entre la cocreación de valor y el valor de la marca de hotel, especialmente si dicho efecto se encadena a la CIM para la sostenibilidad; pues la comunicación efectiva entre los proveedores de servicios y sus clientes ha sido identificada como un antecedente importante para la participación de los clientes en el proceso de cocreación (Auh et al., 2007; Corsaro, 2019) y para el aumento del valor de la marca hotelera (Šerić, Gil-Saura and Ruiz-Molina, 2014).

Además se observa que los consumidores que carecen de conocimientos ecológicos no pueden apreciar las prácticas desarrolladas por los hoteles y confían menos en la reputación medioambiental de la marca (Teng, Lu and Huang, 2018). Sin embargo en el ámbito de la investigación académica son limitados los estudios que tienen por objeto el análisis relativo al conocimiento ecológico por parte del consumidor en el sector hotelero, especialmente cuando su observación se centra en definir si este contribuye o no a aumentar la intensidad de los efectos en las relaciones propuestas mediante el análisis de su rol como moderador (Lin, Lobo and Leckie, 2019; Kushwah, Dhir and Sagar, 2019). En consecuencia, una importante laguna teórica se abre; de ahí que sea una oportunidad de investigación.

De este modo, centrándonos en las empresas turísticas y concretamente en los hoteles, la propuesta que se presenta pretende dar luz al gap identificado en la literatura en las direcciones de análisis expuestas. Así, en primer lugar, evaluamos la influencia de la CIM para la sostenibilidad en la cadena de efectos de cocreación de valor y valor de marca de hotel basado en el cliente. En segundo lugar se propone analizar si en dicha cadena la cocreación de valor puede desempeñar un papel mediador. Por último, se observa el rol que juega el nivel de conocimiento ecológico del huésped sobre las relaciones propuestas entre todas las variables retenidas. Los resultados obtenidos ofrecen información relevante para los gestores de los hoteles, guiándoles en el diseño de estrategias de comunicación con el cliente y de cocreación de valor, anunciando fuentes para la creación de ventajas competitivas relativas al entorno sostenible. En ello radica la importancia y originalidad de la presente investigación. 


\section{Comunicación integrada de marketing y sostenibilidad}

Los últimos años han sido testigos de un renovado interés en el tema de la CIM entre los profesionales y académicos por igual; y su observación, desde el prisma de la sostenibilidad, es una inquietud reciente (Alevizou, Henninger and Spinks, 2019; Bordian, Gil-Saura and Šerić, 2021). Dado que el mercado actual y los consumidores requieren una nueva forma de comunicación que cubra los objetivos de sostenibilidad, esta investigación se propone el análisis del concepto no desde su óptica general sino desde un ángulo más particular relacionado específicamente con el campo sostenible. Así, la CIM para la sostenibilidad es un ámbito de investigación novedoso que abarca tres áreas: cambio climático; responsabilidad social corporativa y consumo sostenible (Bormane, 2018).

Tölkes (2018) constata, a través de su exhaustiva revisión, que la mayoría de los trabajos en el contexto de la CIM para la sostenibilidad consideran únicamente su dimensión medioambiental $(57,4$ $\%)$ y que de forma comparada tan solo el 35,1\% investigan la CIM para la sostenibilidad desde el enfoque más completo que suministra la triple línea base - TBL- a partir de sus tres direcciones de análisis: económica; medioambiental y social. Así pues, con base en la TBL, Bormane (2018) introduce el concepto de CIM para la sostenibilidad; por tanto, señala que es

\footnotetext{
laimplementación de actividades de marketing integrando oportunidades de bienestar público, preservación del medio ambiente y desarrollo económico equilibrado con el fin de aumentar el valor de consumo de un producto o servicio a través de la comunicación de la compañía con los participantes del mercado utilizando canales de distribución. (p. 84)
}

De esta manera la comunicación para la sostenibilidad forma parte de una estrategia de marketing más global que adopta un enfoque integrador y holístico para alcanzar los objetivos corporativos y los objetivos a largo plazo de un desarrollo sostenible (Belz and Peattie, 2012).

\section{Cocreación de valor y la aproximación sostenible}

Durantelosúltimos años, el concepto cocreación de valor ha recibido cada vez más atención por parte de la academia y la profesión. De acuerdo con Vargo y Lusch (2008), a la luz del paradigma del Service-Dominant Logic, el cliente no es el centro de todas las decisiones de la empresa sino que ha de ser concebido como un agente activo más en el proceso de creación de valor. En el marco hotelero, la cocreación de valor se define por Bussery Shulga (2018) como la apreciación de la importancia del servicio por las partes interesadas mediante la evaluación de qué se contribuye y qué se realiza a través de la colaboración.

En el contexto de la sostenibilidad, la cocreación puede ejercer de posible instrumento para desarrollar nuevas innovaciones e introducirlas con éxito en la práctica (Gössling et al., 2009; Arnold, 2017). Chang (2019) propone un concepto original relativo a la cocreación de valor al etiquetarla como 'verde', por lo que la define como "el intercambio activo de ideas ambientales entre una empresa y sus socios y la participación en una o más etapas de producción o consumo para crear valor" (p. 3). No obstante, y dado que hay estudios teóricos y empíricos limitados sobre la cocreación de valor en el contexto de la sostenibilidad (v. g., Arnold (2017) y Chang (2019)), esta área requiere más investigación.

En este estudio la cocreación de valor en el marco de la sostenibilidad es tratada como un concepto multidimensional que consta de diferentes elementos: significancia; colaboración; contribución; reconocimiento y respuesta afectiva; lo anterior, siguiendo la línea de trabajo de Busser y Shulga (2018) que permite mostrar la complejidad del concepto. En esta dirección, la significancia del servicio se estima por el grado de importancia de la actividad en la que el cliente participa y que resulta en un mayor valor cocreado. La colaboración se refiere al valor añadido por parte de todos los actores participantes en el proceso de cocreación. La contribución se entiende como el nivel en que el participante comparte sus propios recursos para lograr los resultados deseados. El reconocimiento contribuye a la valoración positiva de los 
resultados de la cocreación de valor y se refiere al agradecimiento del beneficiario. Por último, la respuesta afectiva representa la parte emocional del participante frente al proceso de cocreación. Cada componente se considera pilar base para la cocreación de valor y ofrece un nuevo enfoque para entender el proceso de cocreación en el ámbito del alojamiento colectivo al considerar que brindando el servicio - incluyendo prácticas sostenibles - la empresa turísticay sus clientes pueden encontrar el proceso significativo y colaborar, contribuir, recibir reconocimiento y generar una respuesta afectiva para cocrear valor verde.

Según Arnold (2017) la cocreación de valor podría generar cambios incrementales hacia el desarrollo de más actividades amigables con el medio ambiente. Por esta razón, la cocreación se torna en una variable crítica que a través de la contribución y la colaboración podría generar una mayor participación de los clientes en las prácticas ecológicas de los hoteles. No obstante, todavía no hay un suficiente número de contribuciones para delimitar con exactitud lo que lleva a los clientes a colaborar y participar en actividades de cocreación de valor relacionadas con las prácticas sostenibles de un hotel. Como se afirma en la literatura (v. g., Auh etal. (2007), Grissemanny Stokburger-Sauer (2012) y Corsaro (2019)), la comunicación efectiva y activa entre los proveedores de servicios y sus clientes es un antecedente importante para la participación de los últimos en el proceso de cocreación de valor. De igual manera algunos trabajos subrayan la importancia de utilizar la comunicación para la sostenibilidad de forma más persuasiva para mejorar la colaboración entre las partes interesadas hacia las prácticas más sostenibles (Font, Elgammal and Lamond, 2017; Tölkes, 2018), concluyendo que esa comunicación persuasiva es capaz de lograr un mayor nivel de colaboración e implicación por parte de los turistas (Han et al., 2018).

No obstante, hasta ahora, no se conocen estudios que hayan investigado el efecto de la CIM sobre la cocreación de valor. Por tanto, según esto, se propone la primera hipótesis:

H1: la CIM para la sostenibilidad tiene un efecto positivo y significativo en la cocreación de valor.

\section{Valor de marca}

El valor de marca desde la perspectiva del consumidor es uno de los conceptos que más atención e interés ha recibido durante los últimos años en la gestión de marketing (Marín-García et al., 2021). De acuerdo con Aaker (1991) el valor de marca se define como "un conjunto de activos y pasivos vinculados a una marca, su nombre y símbolo, que añaden o sustraen valor suministrado por un producto o servicio a una empresa y/o a sus clientes" (p.15). Así, se refleja la idea de que la marca es uno de los activos más importantes de las empresas debido a su capacidad para proporcionar ventajas competitivas exclusivas (Aaker, 1991). De hecho, la incorporación de las prácticas sostenibles en la actividad diaria de la empresa se considera fuente de ventaja competitiva (Lin et al., 2019). Aunque es importante comunicar constantemente dichas ventajas competitivas, así como las iniciativas sostenibles de la empresa para lograr un mayor nivel de reconocimiento y compresión entre sus clientes (Gössling and Buckley, 2016; Wehrli et al., 2014; Tölkes, 2018).

El estudio de Šerić et al. (2014) confirma que el alto nivel de implementación hotelera de la CIM, a partir de la percepción de los huéspedes, puede aumentar el valor de la marca de hotel. Sin embargo todavía se ha avanzado poco en el estudio de esa relación en base ala sostenibilidad; si bien dado que el cliente es cada vez más consciente de la realidad del cambio climático podría esperarse desde la óptica del huésped la ocurrencia de tal efecto. De este modo, y teniendo en cuenta el propósito del presente trabajo, se enuncia la siguiente hipótesis:

H2: la CIM para la sostenibilidad tiene un efecto positivo y significativo en el valor de marca de hotel.

Por otro lado existe una limitada investigación hasta la fecha centrada en la relación entre la cocreación de valor y el valor de marca (v. g., Kristal et al. (2016), González-Mansilla et al. (2019)). En esta, se proporciona evidencia empírica relativa al efecto antecedente de la cocreación en el valor de marca basado en el cliente (Frías-Jamilena, Polo Peña and Rodríguez Molina, 2016). Sobre este supuesto se establece la tercera hipótesis de investigación, bajo el enfoque del desempeño sostenible en la industria hotelera: 
H3: la cocreación de valor tiene un efecto positivo y significativo en el valor de marca de hotel.

\section{Conocimiento ecológico}

El conocimiento ecológico es un elemento crítico en el proceso de toma de decisiones del consumidor (Zhang and Lei, 2012; Teng etal., 2018). De acuerdo con Mostafa (2007) el conocimiento ecológico refleja "qué sabe la gente sobre el medio ambiente, las relaciones clave establecidas con los aspectos o impactos ambientales, una apreciación de todo el sistema ecológico y las responsabilidades colectivas necesarias para el desarrollo sostenible" (p. 221).

Cuando los consumidores no tienen suficiente conocimiento ecológico ellos no pueden apreciar las prácticas llevadas a cabo por los hoteles (Teng et al., 2018) ni, por ende, cocrear valor (Gössling et al., 2009; Arnold, 2017; Chang, 2019). Para Lin et al. (2019) los clientes con bajo nivel de conocimiento ecológico tienen más probabilidades de percibir una mayor incertidumbre, lo que puede aumentar sus actitudes escépticas hacia la marca en términos de sus atributos ecológicos. Por tanto, es menos probable que confíen en la reputación medioambiental de la marca.

El efecto moderador del conocimiento ecológico ha sido investigado recientemente en los trabajos de Lin et al. (2019) y Kushwah et al. (2019), si bien estas aportaciones lo han sido en otros contextos de investigación alejados del sector del alojamiento colectivo. El rol de esta variable sobre la cadena de relaciones observadas en este trabajo es un terreno aún por explorar, por lo que se propone progresar en esta dirección y se plantea la cuarta hipótesis como sigue:

H4a-H4c: las relaciones entre la CIM, la cocreación de valor y el valor de marca varían según el nivel (alto o bajo) del conocimiento ecológico de los huéspedes.

En la figura 1 se muestra el modelo teórico objeto de análisis, el cual representa las relaciones planteadas en forma de hipótesis:

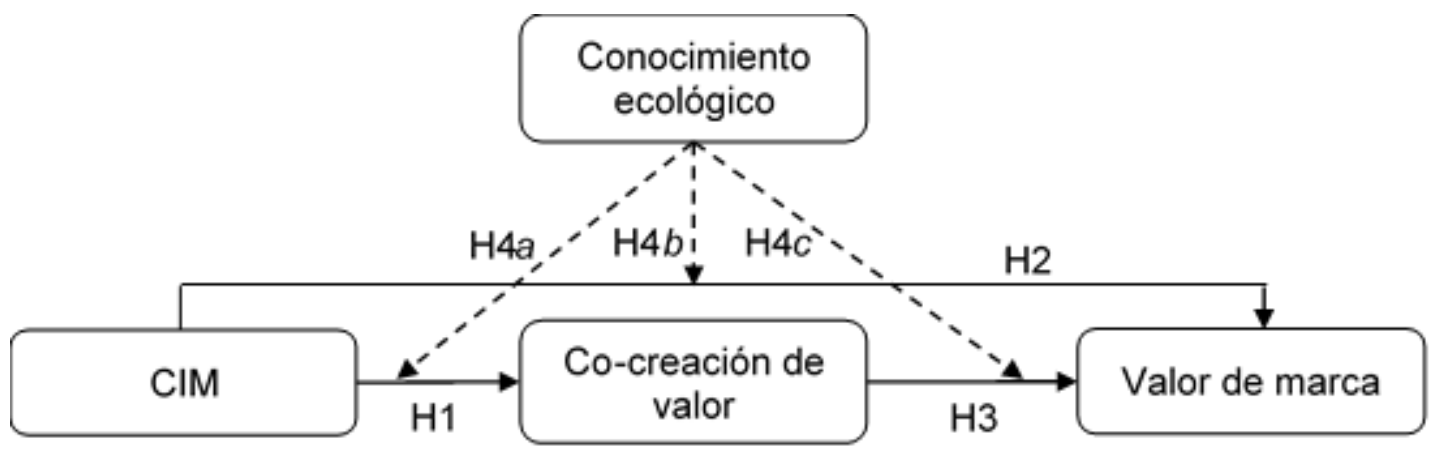

Figura 1. Modelo teórico e hipótesis. Fuente: elaboración propia por parte de las autoras.

\section{Metodología}

Los ítems concernientes a las distintas variables analizadas han sido extraídos y adaptados de diversas escalas utilizadas en la literatura de marketing. Así, todas las escalas se adaptaron para su observación en hoteles y en el marco sostenible. Se utilizaron cuatro ítems relativos a la
CIM, que derivan de la dimensión "comunicaciones unificadas para mensajes e imágenes consistentes" de la escala de medición de Lee y Park (2007). Los ítems incluidos en el cuestionario, relativos a la cocreación de valor, han sido extraídos yadaptados a partir de la escala multidimensional propuesta por Busser y Shulga (2018). Para evaluar el valor de marca se propone retener la escala de carácter unidimensional definida por Christodoulides, Cadogan y Veloutsou (2015). Por último, para 
medir el conocimiento ecológico se retienen siete indicadores extraídos del trabajo de Teng et al. (2018). En todos los casos se utilizaron escalas de medición tipo Likert de siete puntos de anclaje.

Con el objetivo de recoger la información que permitiera contrastar las hipótesis planteadas se procedió a la administración de un cuestionario estructurado ad hoc con respuestas cerradas, en donde el huésped debía indicar su grado de 'acuerdo' o 'desacuerdo' mediante una entrevista personal cara a cara. Para el diseño de la investigación se eligió el procedimiento de muestreo no probabilístico por conveniencia, ya que no se tenía acceso al censo de clientes hoteleros y no era posible determinar la probabilidad de que un elemento de la población debiera pertenecer a la muestra (Trespalacios, Vázquez y Bello, 2005). En total, se obtuvieron 303 cuestionarios válidos; se encuestaron huéspedes que se alojaron en hoteles ubicados en Kiev (Ucrania) durante el mes de agosto de 2018; los clientes fueron interceptados, bien en zonas cercanas del hotel o bien en zonas comerciales de intenso tráfico peatonal en el centro turístico de la ciudad.

Según el perfil demográfico de los encuestados, el $42,9 \%$ fueron hombres y el 57,1 \% mujeres; un $40,9 \%$ de los entrevistados pertenece al segmento de edad entre 18 y 25 años; un 40,3 \% se sitúa entre los 26 y 35 años; un 10,6 \% entre los 36 y 45 años y un 8,2\% más de 46 años. En cuanto a la formación, la mayoría tiene máster $(55,8 \%)$, licenciatura, ingeniería y arquitectura $(26,4 \%)$ como el nivel de estudios principal; mientras que con respecto a su situación laboral un 44,9\% son empleados y un $20,5 \%$ son empresarios. Un $46,5 \%$ eligen alojarse en el hotel $1 \mathrm{vez}$ al año o menos, un 38,6\% entre 2-4 veces al año y un 14,9\% más de 4 veces al año. De los cuales un 28,7 \% estaban alojados solo 1 día en el hotel, un $65 \%$ entre 2-7 días y un 6,3 \% más de 7 días. Por último, un 92,7 \% de los encuestados era de nacionalidad ucraniana y el resto $(7,3 \%)$ de otras nacionalidades.

\section{Resultados}

Una vez determinado el modelo teórico se utilizó la aplicación SmartPLS 3 para estimar el modelo estructural, siguiendo un procedimiento analítico en dos etapas (Hair et al., 2017). En primer lugar se examinó el instrumento de medida mediante el análisis factorial confirmatorio AFC—, comprobando la validez y fiabilidad de las medidas; en segundo lugar se examinó el modelo estructural.

El instrumento de medida para el constructo cocreación de valor como factor de segundo orden propuesto por Busser y Shulga (2018) se estimó utilizando el método de "repeated indicators approach" (Hair et al., 2018). Los resultados del AFC permitieron retener cuatro dimensiones para explicar la cocreación de valor, siendo necesario eliminar la dimensión de 'reconocimiento' por no cumplir con el valor necesario de la relación HT/MT (Henseler, Ringle and Sarstedt, 2015). Los resultados confirmaron la riqueza del concepto al permitir una aproximación multidimensional a la cocreación en términos de significancia, colaboración, contribución y respuesta afectiva.

A partir de los resultados del AFC se afirmó la fiabilidad de todas las escalas, ya que los valores de fiabilidad compuesta y el coeficiente alfa de Cronbach eran superiores a los valores recomendados de 0,7 (Nunnally and Bernstein, 1994) para todos los constructos (tabla 1). Para valorar la validez convergente se llevó a cabo el análisis de las cargas totales estandarizadas, que eran superiores a 0,6 (Hair et al., 2017). Todas las cargas eran estadísticamente significativas para todos los ítems. Además el análisis de varianza extraída -AVE - constató que sus valores superaron el valor crítico de 0,5 establecido en la literatura (Fornell and Larcker, 1981). Todo ello permitió confirmar la fiabilidad de las escalas y la validez convergente de los constructos propuestos. 
MARIIA BORDIAN, IRENE GIL-SAURA, MAJA ŠERIĆ

Tabla 1. Resultados del AFC y propiedades psicométricas del modelo de medida

\begin{tabular}{|c|c|c|c|c|c|c|}
\hline Indicador de medida & & Carga & Valor de $t$ & a de Cron. & IFC & AVE \\
\hline \multirow{4}{*}{ CIM para la sostenibilidad } & $\mathrm{CIM}_{1}$ & $0,828^{\star \star}$ & 36,361 & \multirow{4}{*}{0,851} & \multirow{4}{*}{0,900} & \multirow{4}{*}{0,692} \\
\hline & $\mathrm{CIM}_{2}$ & $0,821^{\star \star}$ & 33,284 & & & \\
\hline & $\mathrm{CIM}_{3}$ & $0,781^{\star \star}$ & 25,301 & & & \\
\hline & $\mathrm{CIM}_{4}$ & $0,892^{\star *}$ & 74,862 & & & \\
\hline \multirow{5}{*}{ Cocreación de valor-significancia } & $\mathrm{CCV}_{1}$ & $0,832^{\star *}$ & 36,650 & \multirow{5}{*}{0,902} & \multirow{5}{*}{0,928} & \multirow{5}{*}{0,720} \\
\hline & $\mathrm{CCV}_{2}$ & $0,841^{\star \star}$ & 44,110 & & & \\
\hline & $\mathrm{CCV}_{3}$ & $0,790^{\star \star}$ & 21,800 & & & \\
\hline & $\mathrm{CCV}_{4}$ & $0,890^{\star *}$ & 64,071 & & & \\
\hline & $\mathrm{CCV}_{5}$ & $0,884^{* *}$ & 52,639 & & & \\
\hline \multirow{5}{*}{ Cocreación de valor-colaboración } & CCV6 & $0,909^{\star \star}$ & 67,679 & \multirow{5}{*}{0,946} & \multirow{5}{*}{0,959} & \multirow{5}{*}{0,823} \\
\hline & $\mathrm{CCV}_{7}$ & $0,905^{\star \star}$ & 37,852 & & & \\
\hline & CCV8 & $0,933^{\star \star}$ & 85,320 & & & \\
\hline & $\mathrm{CCV}_{9}$ & $0,900^{\star \star}$ & 56,439 & & & \\
\hline & $\mathrm{CCV}_{10}$ & $0,889^{\star \star}$ & 49,080 & & & \\
\hline \multirow{5}{*}{ Cocreación de valor-contribución } & $\mathrm{CCV}_{11}$ & $0,827^{\star \star}$ & 30,575 & \multirow{5}{*}{0,933} & \multirow{5}{*}{0,950} & \multirow{5}{*}{0,791} \\
\hline & $\mathrm{CCV}_{12}$ & $0,933^{\star \star}$ & 75,161 & & & \\
\hline & $\mathrm{CCV}_{13}$ & $0,926^{\star \star}$ & 71,072 & & & \\
\hline & $\mathrm{CCV}_{14}$ & $0,894^{\star \star}$ & 50,998 & & & \\
\hline & $\mathrm{CCV}_{15}$ & $0,864^{\star \star}$ & 48,517 & & & \\
\hline \multirow{5}{*}{ Cocreación de valor-respuesta afectiva } & $\mathrm{CCV}_{16}$ & $0,945^{\star \star}$ & 96,357 & \multirow{5}{*}{0,970} & \multirow{5}{*}{0,977} & \multirow{5}{*}{0,894} \\
\hline & $\mathrm{CCV}_{17}$ & $0,963^{\star *}$ & 132,796 & & & \\
\hline & $\mathrm{CCV}_{18}$ & $0,966^{\star \star}$ & 167,397 & & & \\
\hline & $\mathrm{CCV}_{19}$ & $0,961^{\star *}$ & 112,442 & & & \\
\hline & $\mathrm{CCV}_{20}$ & $0,891^{\star *}$ & 44,994 & & & \\
\hline \multirow{12}{*}{ Valor de marca } & $\mathrm{VM}_{1}$ & $0,730 * \star$ & 17,969 & \multirow{12}{*}{0,931} & \multirow{12}{*}{0,940} & \multirow{12}{*}{0,570} \\
\hline & $\mathrm{VM} 2$ & $0,776^{\star \star}$ & 22,537 & & & \\
\hline & $\mathrm{VM}_{3}$ & $0,790^{\star *}$ & 24,404 & & & \\
\hline & $\mathrm{VM}_{4}$ & $0,819^{\star \star}$ & 27,155 & & & \\
\hline & $\mathrm{VM}_{5}$ & $0,828^{\star *}$ & 27,953 & & & \\
\hline & VM6 & $0,823^{\star \star}$ & 43,293 & & & \\
\hline & VM7 & $0,775^{\star \star}$ & 43,412 & & & \\
\hline & VM8 & $0,779^{\star \star}$ & 38,175 & & & \\
\hline & VM9 & $0,773^{\star \star}$ & 32,479 & & & \\
\hline & $\mathrm{VM}_{10}$ & $0,680^{\star *}$ & 20,576 & & & \\
\hline & VM11 & $0,617^{\star \star}$ & 15,481 & & & \\
\hline & $\mathrm{VM}_{12}$ & $0,647^{\star \star}$ & 16,707 & & & \\
\hline
\end{tabular}

Nota: a de Cron.=alpha de Cronbach; IFC=índice de fiabilidad compuesta; AVE=análisis de varianza extraída; ${ }^{\star *} p<0,01 ;{ }^{\star} p<0,05$.

Fuente: elaboración propia por parte de las autoras. 
A continuación, la validez discriminante se estableció a través del AVE (tabla 2) en la que se afirmó que el cuadrado de la correlación calculado entre dos factores era menor que el AVE de cada factor y que ningún indicador tiene una influencia significativa en otro factor que no le corresponde (Fornell and Larcker, 1981). Además la misma regla se confirmó con la relación HT/MT que fue menor que 0,90 para cada factor, lo que significa que las correlaciones de las relaciones entre los indicadores de la misma variable eran mayores que las relaciones entre los indicadores que miden diferentes variables (Henseler et al., 2015).

Tabla 2. Validez discriminante: criterio de Fornell-Larcker y ratio HT/MT

\begin{tabular}{lllllll}
\hline Factor & F1 & F2 & F3 & F4 & F5 & F6 \\
\hline F1. CIM & 0,832 & 0,554 & 0,470 & 0,351 & 0,366 & 0,611 \\
\hline F2. Cocreación de valor-significancia & 0,491 & 0,848 & 0,646 & 0,494 & 0,582 & 0,503 \\
\hline F3. Cocreación de valor-colaboración & 0,427 & 0,603 & 0,907 & 0,791 & 0,718 & 0,360 \\
\hline F4. Cocreación de valor-contribución & 0,315 & 0,462 & 0,742 & 0,890 & 0,792 & 0,277 \\
\hline F5. Cocreación de valor-respuesta afectiva & 0,335 & 0,551 & 0,689 & 0,755 & 0,946 & 0,319 \\
\hline F6. Valor de marca & 0,557 & 0,458 & 0,333 & 0,246 & 0,292 & 0,755 \\
\hline
\end{tabular}

Nota: valores en la diagonal son las raíces cuadradas del AVE; debajo de la diagonal: correlaciones entre los factores; sobre la diagonal: valores de las correlaciones al cuadrado (ratio HT/MT).

Fuente: elaboración propia por parte de las autoras.

Por último, se estimó el modelo estructural a través de mínimos cuadrados parciales - PLSy se comprobó el poder explicativo del modelo a través de los coeficientes de determinación $\mathrm{R}^{2} \mathrm{y}$ los índices de redundancia con validación cruzada $\mathrm{Q}^{2}$. Los valores de $\mathrm{R}^{2}$ superaron el valor de 0,10 (tabla
3), lo que sugiere que la relevancia predictiva del modelo es satisfactoria (Falk and Miller, 1992) y los valores de $\mathrm{Q}^{2}$ eran superiores a 0 ; esto también confirma la relevancia predictiva del modelo (Chin, 1998), lo que nos permitió evaluar la significancia delas relaciones causales previamente establecidas.

Tabla 3. Resultados de la estimación del modelo de ecuaciones estructurales

\begin{tabular}{lllll}
\hline Hipótesis & Relación & $\boldsymbol{\beta}$ (Beta estandarizado) & Valor de t & Contraste \\
\hline $\mathrm{H}_{1}$ & CIM-cocreación de valor & $0,453^{\star *}$ & 9,726 & Aceptada \\
\hline $\mathrm{H}_{2}$ & CIM-valor de marca & $0,481^{\star *}$ & 9,353 & Aceptada \\
\hline $\mathrm{H}_{3}$ & Cocreación de valor-valor de marca & $0,171^{\star *}$ & 3,073 & Aceptada \\
\hline
\end{tabular}

Nota: cocreación de valor: $R^{2}=0,202, Q^{2}=0,117$; valor de marca: $R^{2}=0,331, Q^{2}=0,185$ ** $p<0,01 ; n / s$ : no significativo. 
Los resultados del cálculo indican que se aceptan las tres hipótesis dado que se encuentran relaciones positivas y significativas entre la CIM del hotel para la sostenibilidad y la cocreación de valor $(\beta=0,453, p<0,01 ; \mathrm{H} 1)$ y entre la CIM y el valor de marca de hotel $(\beta=0,481, p<0,01 ; \mathrm{H} 2)$ con valores $t$ respectivos de 9,726 y 9,114 , mostrando una cierta intensidad y fuerza en ambas relaciones. Con respecto a la tercera hipótesis, los hallazgos revelan que el impacto de la cocreación de valor sobre el valor de marca es significativo $(\beta=0,171$, $p<0,01 ; \mathrm{H} 3$ ); aunque de menor intensidad con un valor $t$ de 3,073, lo que lleva a la aceptación de la hipótesis tres.
Por otra parte, los resultados también proporcionan apoyo empírico para la mediación de la cocreación de valor en las relaciones entre la CIM y el valor de marca. Dado que tanto los efectos directos como indirectos entre la CIM y el valor de la marca son significativos se concluyó que la cocreación de valor media parcialmente esta relación. El producto (valor de $p$ ) del efecto directo e indirecto muestra una mediación parcial complementaria, ya que su signo es positivo (Hair et al., 2017). Los resultados obtenidos se muestran en la tabla 4.

Tabla 4. El efecto de la mediación de la cocreación de valor

\begin{tabular}{|c|c|c|c|c|c|c|c|}
\hline \multirow{2}{*}{ Relación } & \multicolumn{2}{|c|}{ Efecto directo } & \multicolumn{2}{|c|}{ Efecto indirecto } & \multicolumn{2}{|c|}{ Efecto total } & \multirow{2}{*}{ Valor de $p$} \\
\hline & $\beta$ & Valor de $t$ & $\beta$ & Valor de $t$ & $\beta$ & Valor de $t$ & \\
\hline $\begin{array}{l}\text { (CIM } \times \text { cocreación de valor) } \\
\text {-> valor de marca }\end{array}$ & 0,481 & $9,353^{\star \star}$ & 0,077 & $3,059^{\star \star}$ & 0,558 & $12,412^{\star \star}$ & 0,037 \\
\hline
\end{tabular}

Nota: ${ }^{\star \star} p<0,01 ; \mathrm{n} / \mathrm{s}$ : no significativo.

Fuente: elaboración propia por parte de las autoras.

Según los resultados el efecto total $\left(\beta_{\text {total }}=0,558\right.$; $t=12,412 ; p<0,01$ ) demuestra que la CIM aumenta no solo el valor de marca de hotel directamente, sino también de forma indirecta a través de la cocreación de valor. Por tanto, el efecto de la CIM en el valor de marca se explica mejor a través la cocreación de valor puesto que al incluir la cocreación en la ecuación los efectos de la CIM para la sostenibilidad se intensifican.

Adicionalmente, de acuerdo con las $\mathrm{H} 4 a-\mathrm{H} 4 c$, se esperó encontrar diferencias significativas en las relaciones entre los constructos en función del nivel de conocimiento ecológico del huésped (alto/bajo). En primer lugar se procedió a dividir la muestra en dos grupos de huéspedes en función de su nivel de conocimiento ecológico. Una vez constatado que la escala de medida de conocimiento ecológico gozaba de la fiabilidad adecuada $(\alpha=0,963, \mathrm{IFC}=0,966$, $\mathrm{AVE}=0,592$ ) se creó un índice de la misma a través del cálculo de la media aritmética de los ítems de la escala. Esta división dio lugar a un grupo de 235 sujetos con alto nivel de conocimiento ecológico y otro de 68 turistas con bajo nivel. Según los resultados de los estadísticos descriptivos se observa la notable diferencia entre las respuestas de los huéspedes con alto y bajo nivel de conocimiento ecológico. Se observa que los huéspedes con alto nivel de conocimiento ecológico han valorado más alto la percepción de la CIM y el valor de marca de los hoteles, comparando con los huéspedes con bajo nivel. También se observa la diferente valoración de la significancia como dimensión de la cocreación entre dos grupos en los que los huéspedes con alto nivel de conocimiento ecológico asignan una significancia más alta al participar en las prácticas ambientales del hotel que los clientes con bajo nivel. Con el fin de comprobar las diferencias que apuntan los estadísticos descriptivos y contrastar las hipótesis relativas al carácter moderador del conocimiento ecológico se llevó a cabo el análisis multigrupo - AMG - de Henseler mediante la aplicación SmartPLS 3. Según Hair et al. (2018) en un AMG los resultados serán significativos al $5 \%$ de probabilidad de nivel de error, si el valor de $p$ es menor que 0,05 o mayor que 0,95 . Los resultados obtenidos se muestran en la tabla 5. 
Tabla 5. Resultados del AMG de Henseler

\begin{tabular}{|c|c|c|c|c|c|c|c|}
\hline \multirow{2}{*}{ Hipótesis } & \multirow{2}{*}{ Relación } & \multicolumn{2}{|c|}{ Grupo 1 alto $C E N=235$} & \multicolumn{2}{|c|}{ Grupo 2 bajo $C E N=68$} & \multicolumn{2}{|c|}{ Grupo 1 frente Grupo 2} \\
\hline & & $\beta$ & Valor de $t$ & $\beta$ & Valor de t & Dif. en $\beta$ & Valor de $p$ \\
\hline $\mathrm{H}_{4} \mathrm{a}$ & CIM-cocreación de valor & 0,414 & $7,621^{\star *}$ & 0,485 & $4,156^{\star \star}$ & $-0,070$ & $0,733 n s$ \\
\hline $\mathrm{H}_{4} b$ & CIM-valor de marca & 0,339 & $5,118^{\star \star}$ & 0,348 & $6,498^{\star *}$ & $-0,320$ & $0,994^{\star}$ \\
\hline $\mathrm{H}_{4} \mathrm{C}$ & Cocreación de valor-valor de marca & 0,209 & $3,118^{\star *}$ & 0,208 & $0,617 \mathrm{~ns}$ & 0,133 & o,17ons \\
\hline
\end{tabular}

Nota: alto CE: cocreación de valor: $R^{2}=0,168, Q^{2}=0,098$; valor de marca: $R^{2}=0,211, Q^{2}=0,105$.

Bajo CE: cocreación de valor: $R^{2}=0,235, Q^{2}=0,109$; valor de marca $R^{2}=0,473, Q^{2}=0,267$.

$\mathrm{CE}$ - conocimiento ecológico; ${ }^{* *} \mathrm{p}<0,01 ;{ }^{*} \mathrm{p}<0,05 ; \mathrm{n} / \mathrm{s}$ : no significativo.

Fuente: elaboración propia por parte de las autoras.

A partir de los resultados AMG obtenidos se observa que (i) las relaciones entre la CIM y la cocreación de valor y (ii) las relaciones entre la cocreación de valor y el valor de marca no tienen diferencias significativas entre dos grupos, por lo que no se aceptan las hipótesis $\mathrm{H} 4 a$ y $\mathrm{H} 4 c$. En cambio, la relación entre la CIM y el valor de marca presenta una conexión más fuerte para la muestra de bajo nivel de conocimiento ecológico que demuestra unos resultados relevantes a la luz de esta investigación y lleva a la aceptación de la hipótesis $\mathrm{H} 4 b$ que permite señalar el efecto moderador en esa relación.

\section{Conclusiones, implicaciones de gestión y líneas futuras de investigación}

Este trabajo contribuye a aumentar el conocimiento en el área de la empresa turística y su gestión de marketing. A partir de los resultados obtenidos, este estudio confirma que el grado en que los hoteles implementan la CIM influye positivamente en la cocreación de valor. Estos hallazgos concuerdan con las conclusiones reportadas en los estudios de Grissemann y Stokburger-Sauer (2012), Han et al. (2018) y Corsaro (2019) que respaldan la afirmación de que la comunicación activa y efectiva impulsa el proceso de cocreación de valor. Además permiten progresar en la riqueza del concepto cocreación en el entorno hotelero al identificar la variable como un concepto que se nutre de diferentes dimensiones. De acuerdo con los resultados obtenidos la cocreación de valor se construye a partir de la significancia, la colaboración, la contribución y la respuesta afectiva. Así que la percepción de CIM que brinda el hotel genera un efecto positivo sobre la importancia del servicio, la cooperación, el intercambio de recursos y la respuesta emocional que en conjunto definen el proceso de cocreación de valor, involucrando al huésped en el desarrollo de prácticas sostenibles del hotel. Por otra parte, la CIM influye significativamente en el valor de marca de hotel; de este modo los resultados se alinean con la contribución de Šerić et al. (2014). Así las cosas, una comunicación para la sostenibilidad integrada y coherente impacta positivamente en las percepciones de los huéspedes sobre el valor de marca de hotel que implementa prácticas sostenibles; lo anterior, pone de manifiesto el rol importante de la CIM al construir un valor de marca favorable de la empresa turística a los ojos de sus clientes.

Asimismo, de manera consistente con el marco teórico, los resultados muestran la influencia significativa de la cocreación de valor en el valor de marca de hotel. El hallazgo se apoya en investigaciones tales como las de Frías-Jamilena et al. (2016) y González-Mansilla et al. (2019); con ello se concluyó que cuánto más alto es el grado de significancia, colaboración, contribución y respuesta afectiva del cliente durante la actividad 
de cocreación de valor (enmarcada en este caso en el desarrollo de prácticas ambientales), más alta es su percepción relativa al valor de marca de hotel.

Por último, y atendiendo al rol que desempeñan la cocreación de valor y el conocimiento ecológico del huésped en los efectos de la CIM sobre el valor de marca, los resultados reportan evidencias interesantes. En primer lugar, en lo relativo al papel de la cocreación de valor, se concluye su carácter mediador en la relación entre la CIM y el valor de marca de hotel; así, se da respuesta a distintas llamadas a la investigación (Berenguer-Contrí et al., 2020). Aunque en la literatura existente hay evidencias reconocidas del impacto de la CIM sobre el valor de marca (v. g., Šerić et al. (2014)), incluir en el análisis el rol mediador de la cocreación aporta interesantes evidencias. Los hallazgos permiten demostrar que el efecto de la CIM en el valor de marca se explica principalmente por la cocreación dado que esta desarrolla un rol de mediador complementario en esta relación, intensificando y favoreciendo la mayor contribución de la CIM sobre el valor de marca de hotel. Asimismo, en lo relativo a los grupos de huéspedes según posean alto o bajo nivel de conocimiento ecológico, se evidencia el efecto de la CIM en el valor de marca de una forma más clara en el caso de los huéspedes con bajo nivel de conocimiento ecológico. Los hallazgos reportan evidencias no alineadas con los resultados de Lin et al. (2019) en el ámbito de la comunicación. Tal vez la diferencia pueda explicarse a la luz del papel determinante mostrado por el conocimiento ecológico cuando no se es un consumidor experto; en este último caso, los mensajes sobre sostenibilidad son recibidos como novedosos y contribuyen a incrementar el valor de marca. Todo ello debe ser tenido en cuenta a la hora de crear la estrategia de comunicación relativa a la sostenibilidad.

Estos resultados permiten plantear un conjunto de implicaciones de gestión. Primero - debido a la creciente tendencia de llevar a cabo prácticas sostenibles y ante la competencia en el sector hotelero- las empresas hoteleras deberán cambiar el enfoque en su estrategia de marketing dela comunicación clásica al evolucionar hacia una estrategia que incluye un enfoque sistémico integrado con el fin de promover la gestión, el pensamiento y la acción sostenible. Como muestran los resultados de este estudio, los huéspedes (especialmente los clientes con el bajo nivel del conocimiento ecológico) tendrán una actitud más positiva hacia la marca de hotel que está implementando prácticas sostenibles y además comunicarán sobre ellas a otros clientes. Por tanto, los gerentes de los hoteles deberán promover el concepto de protección ambiental para aumentar el conocimiento y preocupación ambiental de sus clientes. De ahí que los hoteles que implementan prácticas sostenibles no solo deben usar la publicidad para comunicar sus iniciativas ecológicas sino ocupar un puesto más proactivo al participar en los foros e iniciativas ecológicas, patrocinar programas ambientales y aumentar su presencia en las redes sociales, presentar certificación ecológica y etiquetado ecológico para influir en el conocimiento ecológico de los consumidores y desarrollar una imagen general favorable del hotel.

Segundo - al comprender las diferentes dimensiones de la cocreación de valor- los gerentes pueden determinar qué acciones definen la cocreación de valor durante la prestación del servicio y enfocarse en su mejora con el fin de generar más valor para los clientes y para la propia empresa, especialmente si se considera la cocreación como una ventaja competitiva. No obstante, para mejorar el valor del cliente y la empresa, se necesita un diálogo activo. Para lograr este objetivo es importante crear una comunicación bidireccional entre los huéspedes y los gerentes de los hoteles con los empleados de la empresa, que se muestran como mediadores esenciales, transmitiendo información entre ambas partes. Los hoteles deben apoyar, brindar una comunicación interactiva y facilitar la oportunidad a los huéspedes de colaborar en la cocreación de iniciativas ecológicas a través de la personalización del servicio, el intercambio de experiencias y la discusión de opiniones antes, durante y después de participar en las prácticas sostenibles del hotel, ya que los huéspedes muchas veces desconocen este tipo de actividades y el impacto que su estancia tiene en el medio ambiente. Esto podría lograrse creando una comunidad en línea, utilizando una plataforma atractiva o simplemente a través de la interacción directa entre el huésped y el personal del hotel. En consecuencia, el valor generado durante este proceso de cocreación permitirá a los hoteles obtener mayores ventajas competitivas y aumentar el valor de su marca. 
Además —como los huéspedes a menudo desconocen el impacto que su estancia tiene en el medio ambiente-se recomienda que los gerentes del hotel ayuden a los clientes a comprender cómo pueden minimizar ese impacto (por ejemplo, proporcionando información sobre el tema en las áreas públicas del hotel, en habitaciones y en las páginas Web o comunidades en línea). Eso ayudará a ganar una mayor participación de los huéspedes y también aumentar su consciencia y el compromiso medioambiental. Para recompensar la participación y colaboración de los clientes en prácticas sostenibles, los hoteles podrían implementar descuentos (por ejemplo, por no solicitar toallas nuevas a diario o por no usar productos de tocador provistos por el hotel).

Finalmente cabe apuntar algunas limitaciones del trabajo que podrían considerarse como líneas futuras de investigación. En primer lugar algunas restricciones han debido ser asumidas en cuanto al diseño de la muestra y el alcance geográfico del estudio. En este sentido sería interesante comparar los resultados de este estudio con los resultados obtenidos por una muestra mejor representada recogida en otros emplazamientos geográficos, introduciendo así una variable relacionada con la cultura nacional en el modelo teórico. Además es posible avanzar en esta línea de investigación a partir de la introducción de nuevas variables relevantes en el modelo propuesto tales como la variable satisfacción, WOM, intención de cooperación e intención de estancia en un hotel 'verde' dada la evidencia en la literatura sobre algunas relaciones con otras variables en este contexto de estudio. También sería interesante incluir en el instrumento de medida de valor de marca el concepto sostenibilidad para poder evaluar las percepciones de los clientes de la marca sobre atributos 'verdes' (Chen, 2009). En suma, la investigación futura debería evaluar otros entornos de servicio para aumentar la generalización de los hallazgos.

\section{Referencias}

Aaker, D.A. (1991). Managing brand equity: Capitalizing on the value of a brand name. New York, USA: Free Press.
Alevizou, P., Henninger, C. and Spinks, C. (2019). Communicating Sustainability Practices and Values: A case study approach of a microorganisation in the UK. International Journal of Business and Globalisation, 22(1), 37-52.

Arnold, M. (2017). Fostering sustainability by linking co-creation and relationship management concepts. Journal of Cleaner Production, 140(1), 179-188.

Auh, S. et al. (2007). Co-production and customer loyalty in financial services. Journal of Retailing, 83(3), 359-370.

Belz, F.-M. and Peattie, K. (2012). Sustainability marketing: A global perspective. Chichester, England: Wiley.

Berenguer-Contrí, G. et al. (2020). Value co-creation in B-to-B environments. Journal of Business \& Industrial Marketing, 35(7), 1251-1271.

Bormane, S. (2018). Integrated Marketing Communications in Sustainable Business. Society. Integration. Education. Proceedings of the International Scientific Conference, 6, 80-96.

Bordian, M., Gil-Saura, I. and Šerić, M. (2021). How does Integrated Marketing Commnunication boost guest satisfaction? A proposal through ecological knowledge and value co-creation. Management Letters, 1-14.

Busser, J.A. and Shulga, L.V. (2018). Co-created value: Multidimensional scale and nomological network. Tourism Management, 65, 69-86.

Chang, C.H. (2019). Do green motives influence green product innovation? The mediating role of green value co-creation. Corporate Social Responsibility and Environmental Management, 26(2), 330-340.

Chen, Y.S. (2009). The drivers of green brand equity: Green brand image, green satisfaction, and green trust. Journal of Business Ethics, 93(2), 307-319.

Chin, W.W. (1998). The partial least squares approach to structural equation modelling. En Marcoulides, G.A.(Ed.), Modern methodsfor 
business research (pp. 295-336). New Jersey, USA: Lawrence Erlbaum Associates.

Christodoulides, G., Cadogan, J.W. and Veloutsou, C. (2015). Consumer-based brand equity measurement: lessons learned from an international study. International Marketing Review, 32(3/4), 307-328.

Corsaro, D. (2019). Capturing the broader picture of value co-creation management. European Management Journal, 37(1), 99-116.

Falk, R.F. and Miller, N.B. (1992). A primer for soft modelling. Akron, USA: The University of Akron.

Font, X., Elgammal, I. and Lamond, I. (2017). Greenhushing: the deliberate under communicating of sustainability practices by tourism businesses. Journal of Sustainable Tourism, 25(7), 1007-1023.

Fornell, C. and Larcker, D.F. (1981). Evaluating structural equation models with unobservable variables and measurement error. Journal of Marketing Research, 18(1), 39-50.

Frías-Jamilena, D.M., Polo Peña, A.I. and Rodríguez Molina, M.Á. (2016). The Effect of ValueCreation on Consumer-Based Destination Brand Equity.Journal of Travel Research, 56(8), 1011-1031.

https://doi.org/10.1177/0047287516663650 González-Mansilla, Ó., Berenguer-Contrí, G. and Serra-Cantallops, A. (2019). The impact of value co-creation on hotel brand equity and customer satisfaction. Tourism Management, 75(4), 51-65.

Gössling, S. et al. (2009). Swedish air travelers and voluntary carbon offsets: Towards the cocreation of environmental value? Current Issues in Tourism, 12(1), 1-19.

Gössling, S. and Buckley, R. (2016). Carbon labels in tourism: persuasive communication? Journal of Cleaner Production, 111(Part. B), 358-369.

Grissemann, U.S. and Stokburger-Sauer, N.E. (2012). Customer co-creation of travel services: The role of company support and customer satisfaction with the co-creation performance. Tourism Management, 33(6), 1483-1492.

Hair, J.F. et al. (2017). A primer on partial least squares structural equation modelling (PLSSEM). Thousand Oaks, USA: SAGE.

Hair, J.F. et al. (2018). Advanced issues in partial leastsquares structural equation modeling (PLSSEM). Thousand Oaks, USA: SAGE.

Han, W. et al. (2018). Evaluating user-generated content in social media: an effective approach to encourage greater pro-environmental behaviour in tourism? Journal of Sustainable Tourism, 26(4), 600-614.

Henseler, J., Ringle, C.M. and Sarstedt, M. (2015). A new criterion for assessing discriminant validity in variance-based structural equation modelling. Journal of the Academy of Marketing Science, 43(1), 115-135.

Kim, J., Kim, J. and Marshall, R. (2016). Are two arguments always better than one? European Journal of Marketing, 50(7/8), 1399-1425.

Kristal, S. et al. (2016). Is co-creation really a booster for brand equity? The role of co-creation in observer-based brand equity (OBBE).Journal of Product \& Brand Management, 25(3), 247-262.

Kushwah, S., Dhir, A. and Sagar, M. (2019). Ethical consumption intentions and choice behavior towards organic food. Moderation role of buying and environmental concerns. Journal of Cleaner Production, 236, 1-12.

Lee, D.H. and Park, C.W. (2007). Conceptualization and measurement of multidimensionality of integrated marketing communications. Journal of Advertising Research, 47, 222-236.

Lenzen, M. et al. (2018). The carbon footprint of global tourism. Nature Climate Change, 8(6), 522-528.

Lin, J., Lobo, A. and Leckie, C. (2019). The influence of green brand innovativeness and value perception on brand loyalty: the moderating role of green knowledge. Journal of Strategic Marketing, 27(1), 81-95. 
Marín-García, A. et al. (2021). Sustainability, Store Equity, and Satisfaction: The Moderating Effect of Gender in Retailing. Sustainability, 13(2), 1-20.

Moise, M.S. etal. (2019). Influence of environmental practices on brand equity, satisfaction and word of mouth.Journal of Brand Management, 26(6), 646-657.

Moise, M.S, Gil-Saura, I. and Ruiz-Molina, M.E. (2020). Implications of Value Co-creation in Green Hotels: The Moderating Effect of Trip Purpose and Generational Cohort. Sustainability, 12(23), 1-20.

Mostafa, M.M. (2007). Gender differences in Egyptian consumers' green purchase behaviour: the effects of environmental knowledge, concern, and attitude. International Journal of Consumer Studies, 31(3), 220-229.

Nunnally, J.C. and Bernstein, I.H. (1994). Validity. Psychometric theory.NewYork,USA:McGraw-Hill.

Šerić, M., Gil-Saura, I. and Ruiz-Molina, M.E. (2014). How can integrated marketing communications and advanced technology influence the creation of customer-based brand equity? Evidence from the hospitality industry. International Journal of Hospitality Management, 39, 144-156.

Teng, C.-C., Lu, A.C.C. and Huang, T.-T. (2018). Drivers of consumers' behavioral intention toward green hotels. International Journal of Contemporary Hospitality Management, 30(2), 1134-1151.

Tölkes, C. (2018). Sustainability communication in tourism - A literature review. Tourism Management Perspectives, 27(4), 10-21.

Tölkes, C. (2018). The role of sustainability communication in the attitude-behaviour gap of sustainable tourism. Tourism and Hospitality Research, 20(2), 1-12.

Trespalacios, J.A., Vázquez, R. y Bello, L. (2005). Investigación de mercados. Madrid, España: Thomson.
Vargo, S.L. and Lusch, R.F. (2008). Service-dominant logic: continuing the evolution. Journal of the Academy of Marketing Science, 36(1), 1-10.

Wehrli, R. et al. (2014). How to communicate sustainable tourism products to customers: results from a choice experiment. Current Issues in Tourism, 20(13), 1375-1394.

Zhang, H. and Lei, S.L. (2012). A structural model of residents' intention to participate in ecotourism: The case of a wetland community. Tourism Management, 33(4), 916-925. 